\title{
Double knock-out of Hmga1 and Hipk2 genes causes perinatal death associated to respiratory distress and thyroid abnormalities in mice
}

\author{
Raffaele Gerlini ${ }^{1,3}$, Elena Amendola ${ }^{1}$, Andrea Conte ${ }^{1,4}$, Valeria Valente ${ }^{1}$, Mara Tornincasa ${ }^{1,5}$, Sara Carmela Credendino ${ }^{1}$, \\ Francesca Cammarota', Chiara Gentile', Luigi Di Guida', Simona Paladino', Gabriella De Vita (1) ${ }^{1}$, Alfredo Fusco ${ }^{1,2}$ and \\ Giovanna Maria Pierantoni ${ }^{1}$
}

\begin{abstract}
The serine-threonine kinase homeodomain-interacting protein kinase 2 (HIPK2) modulates important cellular functions during development, acting as a signal integrator of a wide variety of stress signals, and as a regulator of transcription factors and cofactors. We have previously demonstrated that HIPK2 binds and phosphorylates High-Mobility Group A1 (HMGA1), an architectural chromatinic protein ubiquitously expressed in embryonic tissues, decreasing its binding affinity to DNA. To better define the functional role of HIPK2 and HMGA1 interaction in vivo, we generated mice in which both genes are disrupted. About 50\% of these Hmga1/Hipk2 double knock-out (DKO) mice die within $12 \mathrm{~h}$ of life (P1) for respiratory failure. The DKO mice present an altered lung morphology, likely owing to a drastic reduction in the expression of surfactant proteins, that are required for lung development. Consistently, we report that both HMGA1 and HIPK2 proteins positively regulate the transcriptional activity of the genes encoding the surfactant proteins. Moreover, these mice display an altered expression of thyroid differentiation markers, reasonably because of a drastic reduction in the expression of the thyroidspecific transcription factors PAX8 and FOXE1, which we demonstrate here to be positively regulated by HMGA1 and HIPK2. Therefore, these data indicate a critical role of HIPK2/HMGA1 cooperation in lung and thyroid development and function, suggesting the potential involvement of their impairment in the pathogenesis of human lung and thyroid diseases.
\end{abstract}

\section{Introduction}

Homeodomain-interacting protein kinase 2 (HIPK2) belongs to a family of nuclear serine-threonine kinases, originally identified as interactors of homeodomainscontaining corepressor proteins $^{1}$. Several studies have reported the crucial role of HIPK2 in many biological processes from apoptosis to cell proliferation ${ }^{2,3}$. Indeed, it is actively involved in cytokinesis, transcriptional regulation, signal transduction, and regulation of protein stability (reviewed in ref. ${ }^{2-5}$ ). Generation of Hipk2 null mice (K2-

\footnotetext{
Correspondence: Alfredo Fusco (alfusco@unina.it) or Giovanna

Maria Pierantoni (gmpieran@unina.it)

${ }^{1}$ Department of Molecular Medicine and Medical Biotechnology, University of Naples, Naples, Italy

${ }^{2}$ Institute of Endocrinology and Experimental Oncology, CNR, Naples, Italy

Full list of author information is available at the end of the article.

Edited by S. Soddu
}

$\mathrm{KO})$ has been reported: they are significantly smaller than their wild-type littermates (WT) and show several neuronal defects $^{6}$, including a reduction of midbrain dopamine neuron survival ${ }^{7}$ and apoptosis of cerebellar Purkinje cells, associated to several psychomotor behavioral abnormalities ${ }^{8}$.

The ability of HIPK2 to bind and phosphorylate several chromatin modifiers and transcription factors likely accounts for the wide spectrum of its biological functions. We have previously identified the nuclear non-histone chromatin high-mobility group A1 proteins as HIPK2 interactors and substrates ${ }^{9}$. HMGA1a and HMGA1b are encoded by the same gene (HMGA1) by alternative splicing. They belong to the HMGA protein family together with HMGA2, which is encoded by a different gene ${ }^{10,11}$. HMGA proteins are able to regulate the expression of several genes by binding to the minor groove of AT-rich DNA sequences

\section{(c) The Author(s) 2019}

(c) (i) Open Access This article is licensed under a Creative Commons Attribution 4.0 International License, which permits use, sharing, adaptation, distribution and reproduction in any medium or format, as long as you give appropriate credit to the original author(s) and the source, provide a link to the Creative Commons license, and indicate if changes were made. The images or other third party material in this article are included in the article's Creative Commons license, unless indicated otherwise in a credit line to the material. If material is not included in the article's Creative Commons license and your intended use is not permitted by statutory regulation or exceeds the permitted use, you will need to obtain permission directly from the copyright holder. To view a copy of this license, visit http://creativecommons.org/licenses/by/4.0/. 
and alter the chromatin structure through the interaction with several transcription factors ${ }^{12}$. HMGA proteins are abundantly expressed during embryogenesis and in most benign ${ }^{13,14}$ and malignant cancer tissues ${ }^{12}$, whereas they are expressed at low levels in the normal adult ones ${ }^{15,16}$. HMGA proteins have a well-established oncogenic activity, and usually their expression correlates with a highly malignant phenotype $^{12}$. HMGA1 controls a wide spectrum of cell functions, including cell proliferation and survival ${ }^{17}$, genomic stability ${ }^{18,19}$, and autophagy ${ }^{20}$. During development, HMGA proteins are involved also in the regulation of body size, as demonstrated by the observation of the "superpygmy" phenotype of the Hmga1/Hmga2 double knock-out (DKO) mice $^{21}$. Moreover, the biological activities of the HMGA proteins are highly regulated by their posttranslational modifications, such as acetylation, methylation, and phosphorylation, which have been associated with cellular transformation and proliferation ${ }^{22}$.

Several studies evidence that HMGA1 and HIPK2 share various fields of interactions, such as regulation of cell proliferation, apoptosis, and p53 activity. Indeed, whereas HMGA1 is able to antagonize p53-driven transcription of apoptosis-related genes, HIPK2 instead is able to potentiate p53 pro-apoptotic activity by phosphorylating its Ser $46^{2,12}$. As evidence of their interaction, it has been reported that HIPK2 phosphorylates HMGA1a at Ser-35, Thr-52, and Thr-77 residues and its isoform HMGA1b at the corresponding sites Thr- 41 and Thr-66, decreasing their binding affinity to DNA and altering the HMGA1mediated regulation of gene expression ${ }^{23,24}$.

On this basis, to understand the role of HMGA1/HIPK2 functional interaction in vivo, we generated mice carrying the disruption of both the Hmgal and Hipk2 genes by crossing the HMGA1-KO(A1-KO) with the K2-KO mice $^{8,21,25}$. Here, we report that Hmga1/Hipk2 DKO mice die within $12 \mathrm{~h}$ of life for respiratory failure, likely owing to impaired lung development associated to a drastic reduction in surfactant proteins, whose expression is positively regulated by both HMGA1 and HIPK2. Moreover, Hmga1/Hipk2 DKO mice show also a reduced expression of thyroid differentiation markers consequent to the drastic downregulation of two transcription factors required for thyroid differentiation, namely PAX8 (paired box gene 8) and FOXE1 (formerly called TTF-2 for Thyroid Transcription Factor-2).

\section{Results}

\section{Generation of Hmga1/Hipk2 DKO}

To generate Hmga1/Hipk2 DKO, we crossed A1-KO mice with $\mathrm{K} 2-\mathrm{KO}$ mice, generating $\mathrm{Hmgal}^{+/-} / \mathrm{Hipk}^{+/-}$ mice, that were then mated, obtaining several combinations of Hmga1 and Hipk2 null alleles, including DKO. We verified the lack of Hmga1 and Hipk2 expression in the DKO mice at mRNA and protein level by RT-PCR and
Western blot, respectively. As shown in Fig. 1a, b, no Hmgal expression was detected in mouse embryo fibroblasts (MEFs), lung, and spleen from DKO and A1-KO mice. Equally, Hipk2 was not expressed in the MEFs, lung and kidney from DKO and K2-KO mice (Fig. 1a, b). Conversely, Hmga1 and Hipk2 have been found expressed in the same MEFs and tissues of the control WT mice.

\section{Newborn DKO mice show respiratory failure}

Single Hmgal and single Hipk2 KO mice were alive, fertile, and developed normally, as previously demonstrated $^{6,8,21}$. Conversely, 29 out of 58 (50\%) DKO mice from different litters were cyanotic at birth, showing breathing difficulties, and did not survive more than $12 \mathrm{~h}$ (P1, Fig. 2a). However, the remaining 50\% fraction did not show any sign of respiratory failure and survived. They developed normally but with a significant lower body weight (for both males and females), until the 17th week of life when they finally catch-up with their WT counterpart (Fig. 2b, c and Supplementary Fig. 1).

To identify the reasons accounting for the cyanotic appearance and breathing dysfunction of the DKO mice which showed perinatal death, we performed a post mortem histological pulmonary examination. As shown in Fig. 2d, most of the lungs, in which Hmgal and Hipk2 were absent, were characterized by the presence of collapsed, immature, sac-like alveoli with unexpanded alveolar space. This condition, known as neonatal atelectasis, is lined by immature cuboidal pneumocytes and characterized by abundant floccular eosinophilic material typical of an incorrect pulmonary development. Moreover, the observed atelectasis was often associated with prominent vasoconstriction of peribronchiolar arterioles (Fig. 2d) probably elicited by sustained hypoxia. As expected, no lung abnormalities were detected either in WT, $A 1-\mathrm{KO}$, and $K 2-\mathrm{KO}$ mice (Supplementary Fig. 2A), or in the DKO mice which do not display respiratory distress (Supplementary Fig. 2b). According to the criteria of mouse embryo development stages described by "Theiler" in "The House Mouse: Atlas of Mouse Development", the immature pulmonary pattern of newborn DKO mice is consistent with the earlier Theiler's stages 25/26 of development, whereas WT mice show Theiler's stage 27 at birth.

Therefore, the lack of both Hmga1 and Hipk2 results in high neonatal mortality, caused by the inability of the lungs to initiate or complete respiration after birth.

\section{HMGA1 and HIPK2 proteins regulate the expression of surfactant proteins}

The expression of genes encoding surfactant proteins (SP-A, SP-B, and SP-C) is limited to the pulmonary epithelial cells and is fundamental for their identity and maturation. The impairment of the regulation of such 


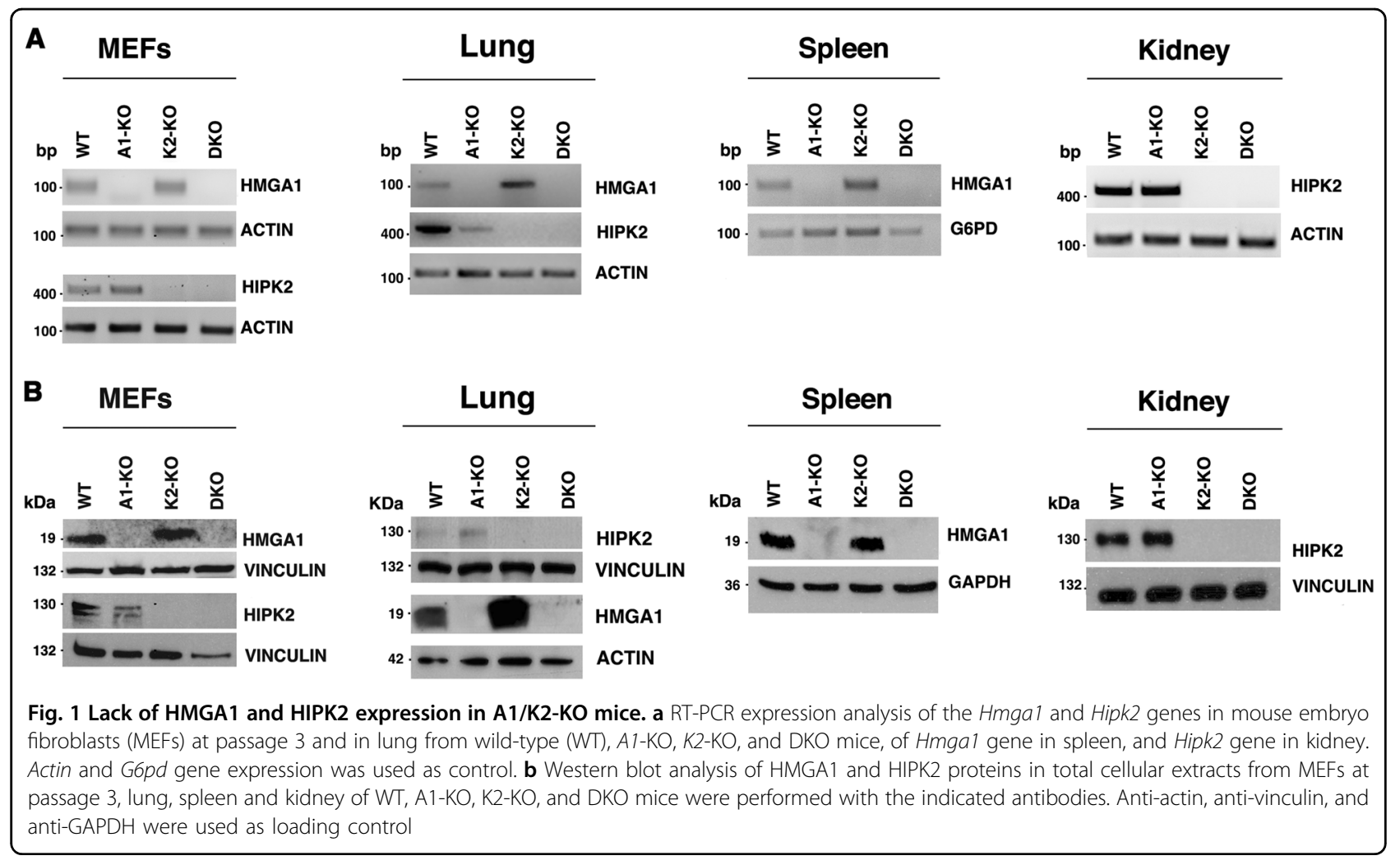

genes results in lethal neonatal respiratory distress in mice and humans (reviewed in ref. ${ }^{26}$ ). During organogenesis, the earliest surfactant protein of the pre-type II cells is SP$\mathrm{C}$ that, being expressed exclusively in the alveolar type II cells, is considered the most specific marker of appropriate alveolar maturation and successful remodeling of the lung (reviewed in ref. ${ }^{27}$ ).

For these reasons, we checked the expression levels of surfactant genes in lungs of DKO mice, which showed perinatal death at $\mathrm{P} 1^{26}$, by quantitative PCR (qPCR). We found that $S p-A$ and $S p-B$ expression was almost undetectable in all the analyzed DKO mice, whereas $S p-C$ expression was strongly decreased $(\sim 58 \%)$ in DKO mice with respect to their WT counterpart (Fig. 3a). Consistently, protein levels of surfactant protein $\mathrm{C}$ (SP-C) in lungs from DKO were lower than in lungs from WT mice at birth, as shown by western blot (Fig. 3b) and immunohistochemical (IHC) analyses (Fig. 3c and Supplemental Fig. S3). Noteworthy, surfactant proteins expression does not change in $A 1-\mathrm{KO}$ and $\mathrm{K} 2-\mathrm{KO}$ mice with respect to WT ones, as demonstrated by western blotting and IHC experiments (Supplementary Fig. 3). Moreover, accordingly also with the expression of Hmga1 and Hipk2 at E17.5 ${ }^{27,28}$, a reduction of $\sim 50 \%$ of $S p-A$, $S p-B$, and $S p-C$ expression was found in lungs from DKO fetuses at E17.5, that normally express $S p-A, S p-B$, and $S p-C$ (Fig. 4a, b), and do not change in $A 1-\mathrm{KO}$ and $K 2-\mathrm{KO}$ mice (Supplementary Fig. 4), indicating that their expression decreases during embryonic development ${ }^{29-31}$.

Because of the observed impairment of the surfactant protein expression in the lungs of DKO fetuses and newborns, we wondered whether HMGA1 and HIPK2 together are able to regulate the $S p-C$ promoter. To this purpose, a luciferase assay activity was performed in HeLa cells, already used to study the regulation of $\mathrm{Sp}-\mathrm{C}$ promoter $^{32}$, in which an expression vector encoding the transactivator NKX2.1 (not endogenously expressed but required for the $S p-C$ transcription) has been transfected together with HMGA1 and/or HIPK2-coding plasmids. As shown in Fig. 4c, both HMGA1 and HIPK2 significantly increased $S p$-C promoter activity in comparison with control-transfected cells. Consistently with a cooperative action of HMGA1 and HIPK2, the overexpression of both proteins resulted in a more drastic increase of $S p-C$ promoter activity. Furthermore, to verify whether HMGA1 and HIPK2 silencing affects the expression of surfactant proteins also in vitro, we knocked down their expression with specific siRNA in the human bronchial epithelial cell line BEAS-2B. However, as shown in the Fig. $4 \mathrm{~d}$, there are no changes in the expression levels of surfactant protein after the interference of HMGA1 and/ or HIPK2 in BEAS-2B cells. These results seem to indicate that the mechanisms regulating surfactant protein expression in vitro are different from those ones operating in vivo. 


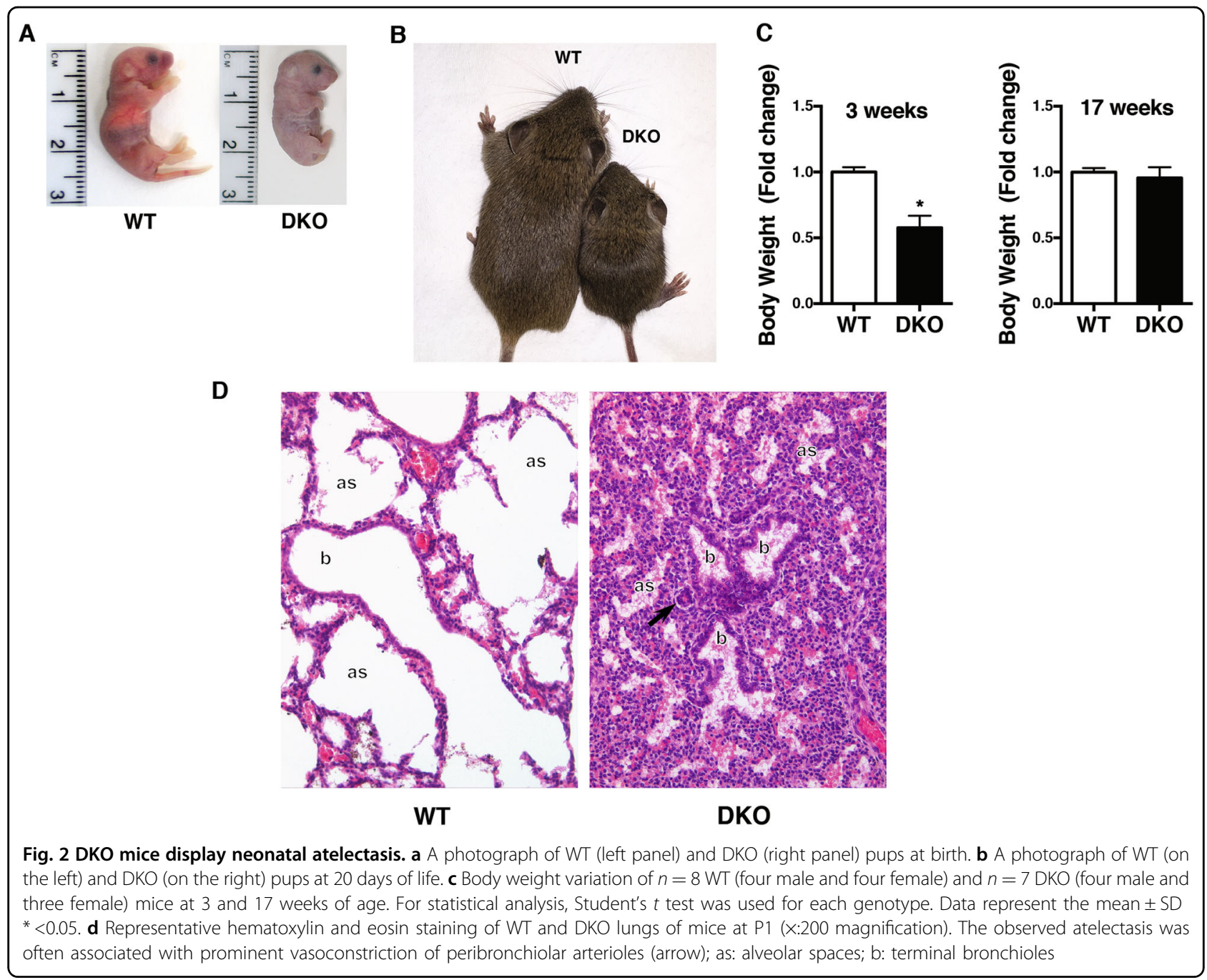

Since NKX2.1 (formerly called TTF-1 for Thyroid Transcription Factor-1), a member of the NKX2 family of homeodomain-containing transcription factors, is required for lung morphogenesis ${ }^{33}$ and the transcription of the surfactant genes ${ }^{32,34-36}$, we evaluated whether the lung defects found in DKO mice were associated with an impairment of $N K X 2.1$ expression. To this aim, we performed real-time qPCR and IHC analysis on lungs from WT and DKO mice at E17.5 and at P1 (Fig. 5a-d). We found that NKX2.1 level did not change either at E17.5 or at $\mathrm{P} 1$, suggesting that the strong reduction of surfactant expression in lungs of DKO mice was not dependent on a reduced $N k \times 2.1$ expression. Furthermore, $N k x 2.1$ expression levels are not affected in $A 1-\mathrm{KO}$ and $\mathrm{K} 2-\mathrm{KO}$ mice (Supplementary Fig. 5).

According to these results, the proper expression of surfactant genes requires in vivo the presence of both the HMGA1 and HIPK2 proteins, and their lack mainly accounts for the atelectasis condition with a respiratory failure of DKO mice at birth.

\section{Impairment of thyroid in DKO mice}

The autoptic examination of thyroid glands of P1 DKO mice which showed the lung phenotype revealed also thyroid abnormalities. Indeed, thyroid follicles showed an irregular structure and were devoid of colloid, in contrast with the WT littermates (Fig. 6a, b). Then, we investigated the expression of the transcription factors which are required for the expression of the thyroid specific differentiation markers. In particular, we analyzed NKX2.1 by IHC (Fig. 6c, d), FOXE1 by IHC (Fig. 6e, f), and PAX8 by both IHC and qPCR (Fig. 6g, h, and k). FOXE1 and PAX8, but not NKX2.1, expression levels were strongly reduced in thyroids from DKO with respect to their WT counterpart. IHC and molecular analysis of late differentiation markers on DKO thyroid glands showed a reduction of expression levels of Thyroglobulin (TG) mRNA (Fig. 6k) and protein, which remained mainly localized at the periphery of the gland (Fig. 6i, j). The expression of other typical genes of differentiated thyroid follicular cells revealed that also Tireoperoxidase (Tpo) and Thyrotropin 
A
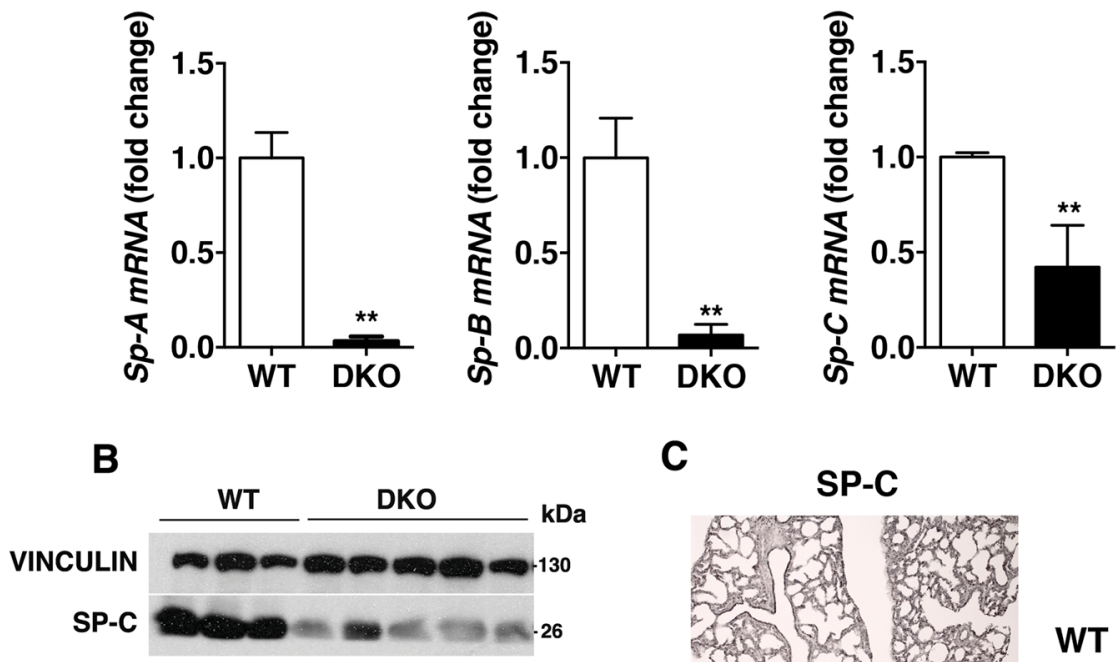

C
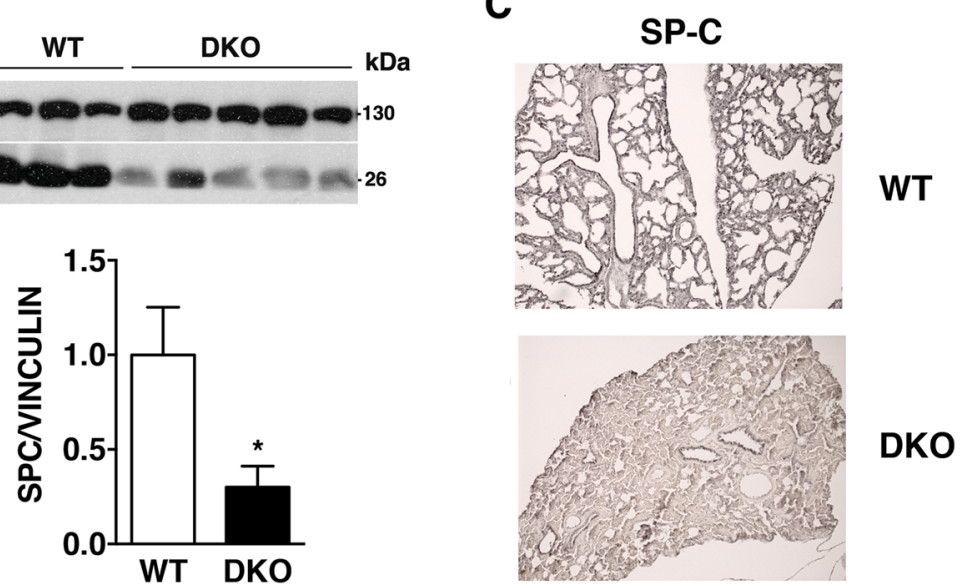

Fig. 3 Depletion of HMGA1 and HIPK2 causes a drastic reduction of Surfactant expression levels. a RNA extracted from lungs of WT and DKO mice at P1 were analyzed by qRT-PCR for Sp-A, Sp-B, and Sp-C expression. The Actin expression level has been used for normalization. $n=3$ WT mice and $n=10$ DKO mice were analyzed. Data are mean \pm SD of a representative experiment performed in triplicate; ${ }^{*} P<0.05$, ${ }^{* *} P<0.01$ (Student's $t$ test). $\mathbf{b}$ Analysis of SP-C protein levels by western blot experiments and relative densitometry from proteins extracted from lungs of $n=3$ WT and $n=5$ DKO mice. Vinculin was used for normalization. c Immunohistochemistry analysis of SP-C at P1 WT versus DKO lung (100x magnification). One representative experiment is shown

receptor (Tshr) mRNA was significantly reduced in DKO mice compared with the control mice (Fig. 6k). No abnormalities were detected in the morphology and in the IHC staining of NKX2.1, FOXE1, PAX8, and TG of single KO thyroids (Supplementary Fig. S6).

These data suggest that lack of both Hmga1 and Hipk2 genes impairs the expression of PAX8 and FOXE1 in thyroid gland and, consequently, of thyroid differentiation markers.

\section{Discussion}

The present study unravels an important role of HIPK2/ HMGA1 functional interaction in determining neonatal survival, lung maturation, and thyroid development. Indeed, $\sim 50 \%$ of HIPK2/HMGA1 DKO mice display perinatal lethality associated to respiratory distress and thyroid abnormalities. In particular, absence of both HIPK2 and HMGA1 expression resulted in impaired lung and thyroid development. In fact, the maturation of the lung alveolar epithelium is delayed in the DKO mice, as indicated by post-mortem analysis showing collapsed immature sac-like alveoli and atelectasis (Fig. 2d), then compromising a correct lung development. Noteworthy, both $A 1-\mathrm{KO}$ and $\mathrm{K} 2-\mathrm{KO}$ mice were generated in a mixed genetic background C57BL6/Sv129J. Therefore, we retain that the heterogeneity of the DKO genotype may account for the different survival observed among litters and among pups of the same litter. It is likely that modifier genes affect the development of lung and thyroid pathology in DKO mice.

On the basis of the results reported here, it is likely that the impairment of the lung function is due to the ability of HIPK2 and HMGA1 to regulate the transcription of genes coding for surfactant proteins. In fact, surfactant factors are involved in the formation of a protein-lipid complex, which, by reducing alveolar surface tension, facilitates the processes that are impaired in DKO mice, such as alveolar space opening, lung fluid clearance, and inflation at birth $^{37-39}$. Consistently with this hypothesis, we report that in the DKO mice which displayed the lung pathologic 
A

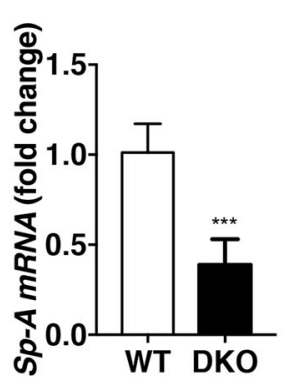

B

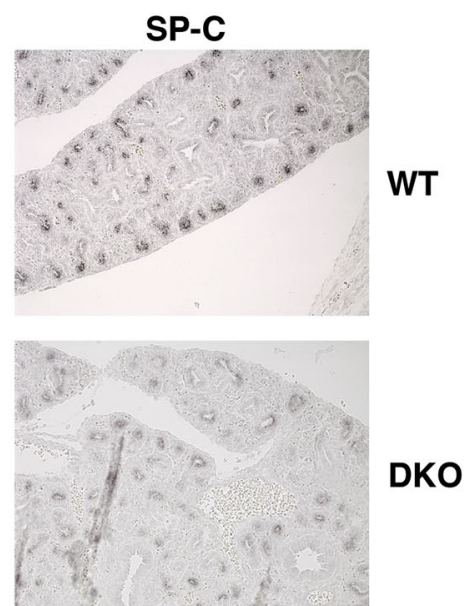

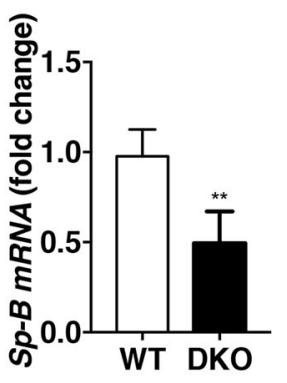
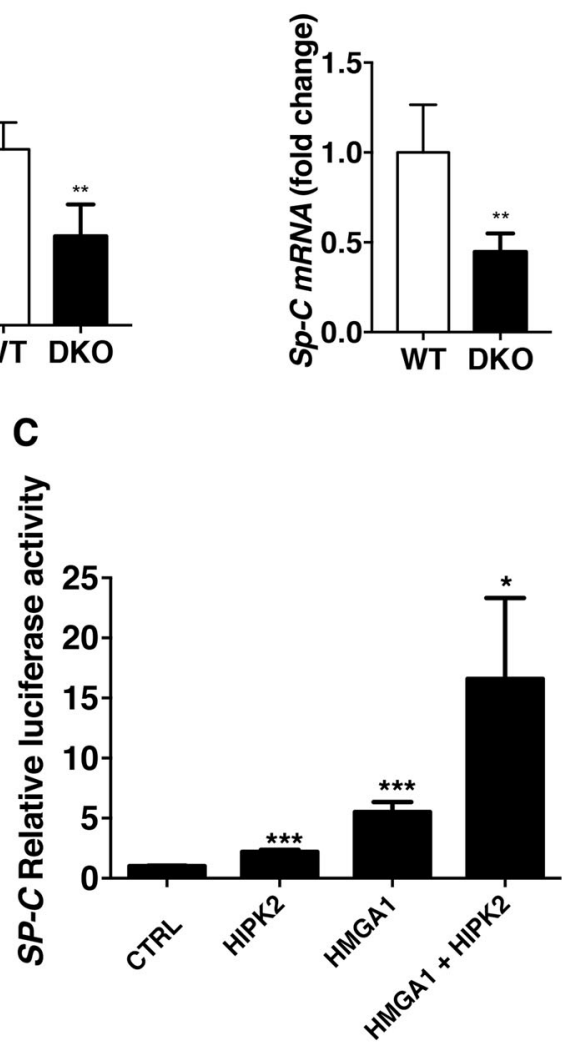

D

BEAS-2B
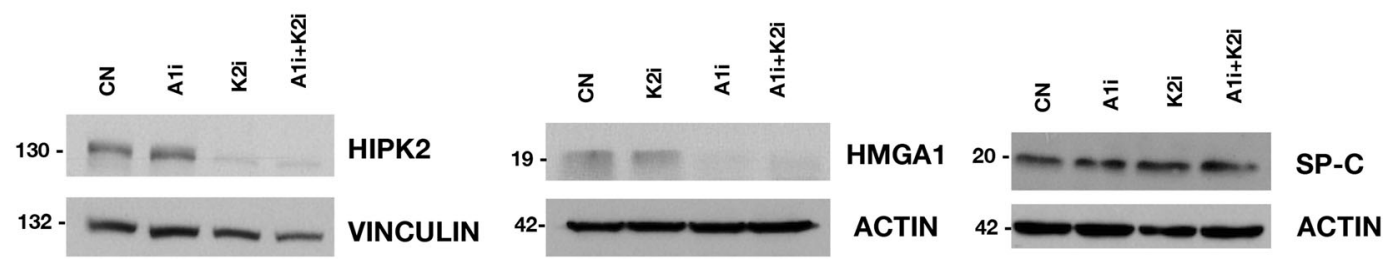

Fig. 4 Surfactant expression is reduced during embryogenesis in DKO mice and is regulated by HMGA1 and HIPK2 expression in cells. a RNA extracted from lungs of WT and DKO mice at E17.5 d.p.c. was analyzed by qRT-PCR for Sp-A, Sp-B, and Sp-C expression. The Actin expression level has been used for normalization. Data are mean \pm SD of a representative experiment performed in triplicate; ${ }^{*}$ indicates significant difference $P<0.01$, ${ }^{* * *} P<0.001 . n=3$ WT mice and $n=10$ DKO mice were analyzed. $\mathbf{b}$ Immunohistochemistry for SP-C on WT and DKO embryos ( $\times 200$ magnification). One representative picture is shown. $\mathbf{c}$ HeLa cells were transfected with Nkx2.1 expression vector alone (Ctrl) or with the indicated plasmids. All transfections were performed in triplicate. Data are mean \pm SD of a representative experiment performed in triplicate; ${ }^{*}$ indicates significant difference $P<0.05,{ }^{* *} P<0.01,{ }^{* * *} P<0.001$ (Student's $t$ test). d BEAS-2B cells were transfected with control (CN), anti-HMGA1 (A1i), anti-HIPK2 (K2i), or anti-HMGA1 and anti-HIPK2 (A1i + K2i), and, after $72 \mathrm{~h}$, proteins were extracted to perform western blot analysis with the indicated antibodies. Antiactin and anti-vinculin antibodies were used as loading control

phenotype, $S p-A$ and $S p-B$ expression was almost undetectable and $S p-C$ expression was strongly decreased ( $58 \%)$ with respect to their WT counterpart (Fig. 3 and Supplemental S3), and that this is associated to the delayed maturation of pulmonary epithelial cells. Moreover, we demonstrate that the activity of the $S p$ - $C$ promoter is regulated by both the HMGA1 and HIPK2 proteins, thus strongly suggesting that the drastic reduction of surfactant expression caused by the loss of HMGA1 and HIPK2 expression is responsible for the pathogenesis of respiratory distress. SP-A, B, C, and D proteins make the $10 \%$ of the complex and their expression is selectively confined to the respiratory epithelium of the lung ${ }^{40}$. Interestingly, HMGA1 and HIPK2 in vitro silencing fails to reproduce the effects of Hmgal and Hipk2 in vivo knock-out on surfactant proteins expression 
A

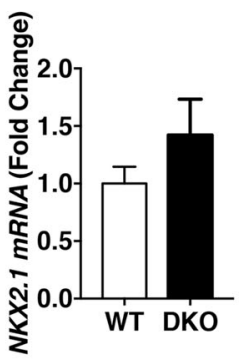

B

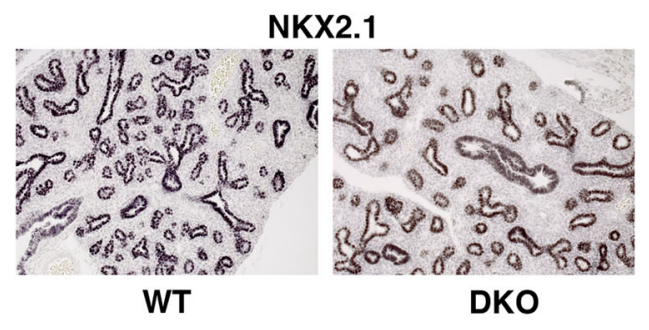

P1

C

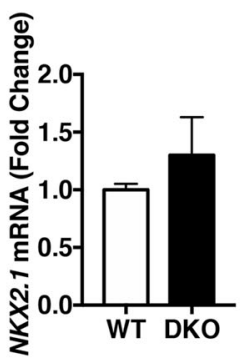

D

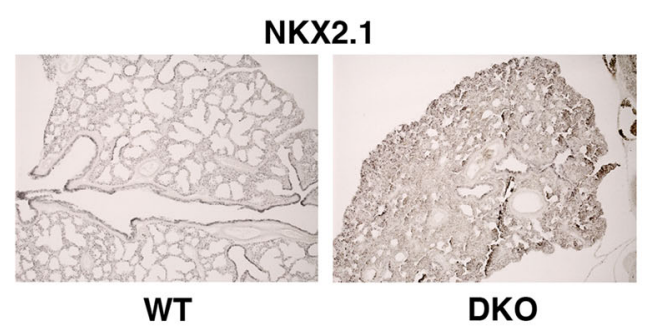

Fig. 5 Hmga1 and Hipk2 deletion does not affect NKX2.1 expression levels. a The same RNAs used in Fig. 4a were analyzed for Nkx2.1 expression by qRT-PCR. $\mathbf{b}$ Immunohistochemistry for NKX2.1 at E17.5 d.p.c. WT versus DKO lung (× 200 magnification). One representative experiment is shown. $\mathbf{c}$ The same RNAs used in Fig. 3a were analyzed for Nkx2.1 expression by qRT-PCR. $\mathbf{d}$ Immunohistochemistry for NKX2.1 at P1 WT versus DKO lung ( $\times 100$ magnification). One representative experiment is shown

in the human bronchial epithelial cell line BEAS-2B. This discrepancy between in vitro and in vivo data is not surprising, and can have multiple explanations: (I) the effects of HMGA1 and/or HIPK2 depletion on surfactant expression might require the interaction with other cell types (non-cell-autonomous effects) and/or the presence specific components of the extra-cellular environment which are not present in the in vitro system; (II) the human model represents terminally differentiated cells, in which different mechanisms may regulate surfactant genes expression. Therefore, the in vitro model might not be suitable to reproduce the observations taken in vivo. According to this hypothesis, it has been already reported that in vitro data do not always reproduce in vivo observations ${ }^{41}$. The discrepancy between in vitro and in vivo data reported here points out the limitation of in vitro studies then underlining the need of studying the functional interaction of HMGA1 and HIPK2 in vivo.

It is established that disruption of the lipid complex, inability of surfactant relocation in the alveolar type II cell, and altered expression of SP-B and SP-C proteins could lead to severe respiratory distress in newborn infants and in mice ${ }^{42}$. These alterations compromise the function of the lung, which may, in turn, lead to lung atelectasis. It is noteworthy that mutations in the SFTPB human gene are associated with fatal respiratory distress in the neonatal period, and mutations in the SFTPC gene are more commonly associated with interstitial lung disease in older infants, children, and adults ${ }^{43}$. Moreover, it has been previously reported that HIPK2 regulates the hypoxiainducible factor-1a (HIF-1a $)^{44}$ that is responsible, among other transcription factors, for fetal lung development as it allows the fetus to quickly adapt to varying oxygen concentrations. Then, lung-specific HIF-1 $\alpha \mathrm{KO}$ leads to perinatal death in mice associated to impaired alveolar epithelial differentiation with consequent loss of surfactant protein expression ${ }^{45}$. Therefore, the loss of HIPK2 expression may affect surfactant levels also by modulating HIF-1 $\alpha$. However, the hypothesis that the loss of Hmgal and Hipk2 could affect lung development by some other mechanisms, independent from their ability to activate surfactant protein expression, should be taken in consideration, and may be further investigated. In this perspective, it is known that mice carrying the impairment of both Ulk1 and Ulk2 genes show a pulmonary phenotype similar to the DKO mice, including perinatal respiratory distress and lethality, however in absence of a decreased surfactant expression ${ }^{46}$. The impairment of the 


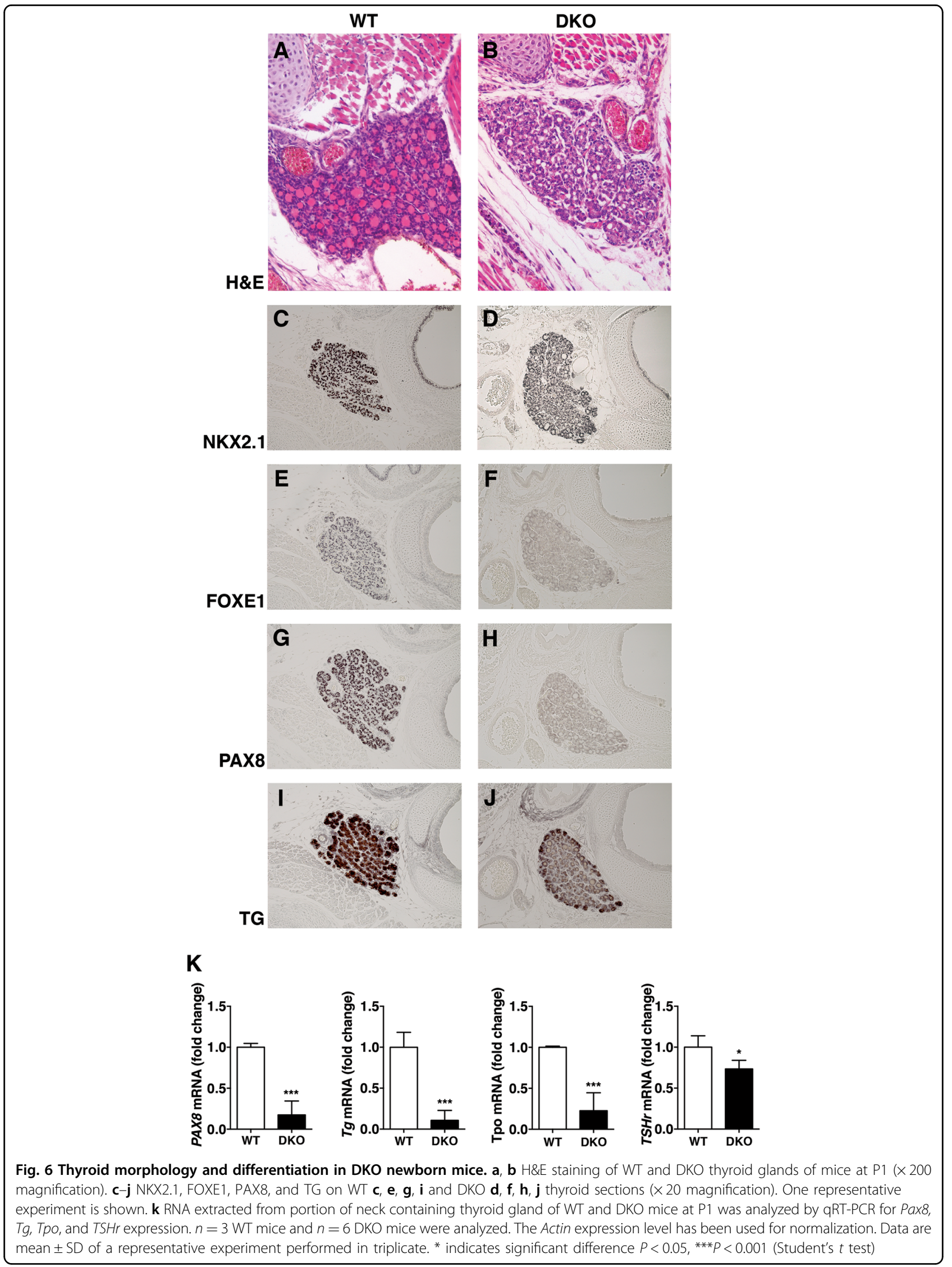


autophagic functions seems to account for the pathology shown by these mice. Then, it is noteworthy that we have recently reported that HMGA1 regulates autophagy by repressing the expression of the Ulk1 and Ulk2 genes ${ }^{20}$.

In addition to the lung phenotype, Hmga 1/Hipk2 DKO mice show other phenotypic features related to the development of the thyroid, in terms of lack of colloid accumulation in thyroid follicles and failure of the normal thyroid differentiation. Indeed, the specific markers of thyroid differentiation such as TPO, TSHr, and TG are downregulated in DKO mice when compared with the WT, with the TG relocated mostly at the margins of the gland. The reduced expression of two thyroid specific transcription factors TTF-2 and PAX8, that have been already described as crucial for thyroid development and differentiation ${ }^{47,48}$, likely accounts for this phenotype.

The association between lung and thyroid dysfunctions is not surprising as both organs derive from the same embryonic layer, the endoderm. Moreover, during the perinatal period, adequate levels of thyroid hormones are critical for promoting proper lung maturation. Consistently, the correct development of lung and thyroid involves one common player, the transcription factor NKX2-1 ${ }^{33}$. Therefore, we have also investigated the possible involvement of NKX2.1 (also called TTF-1) in the thyroid pathology of the DKO mice. In fact, NKX2.1 is essential for brain, thyroid, and lung differentiation and development $^{49}$ and, consistently, it regulates TG, TPO, and TSHr gene expression ${ }^{50-52}$. Moreover, it is required for Sftpc, Sftpa, Sftpb, and its absence causes lack of the lung parenchyma ${ }^{34-36,53,54}$. Surprisingly, we did not observe any difference in the levels of NKX2.1 expression in lungs and thyroids, between WT and DKO, both at birth and during embryogenesis (Fig. 5 and Supplementary Fig. 5). However, we cannot rule out the hypothesis that HMGA1 and HIPK2 might modulate NKX2.1 function by acting at post-translational level impairing its phosphorylation that is critical for NKX2.1 transcriptional activity $^{55,56}$

In conclusion, the results reported here indicate a new critical role of the HIPK2/HMGA1 functional interaction in lung and thyroid development and homeostasis. Then, the involvement of possible alterations of both these genes might be investigated in diseases affecting lung and thyroid development.

\section{Materials and methods}

Generation of $\mathrm{Hmga1}^{-/-} / \mathrm{Hipk2}^{-/-}$mice, genotyping and primary MEFs

The Hmga1 ${ }^{-l-} /$ Hipk2 $^{-1-}$ mice (DKO) were generated by crossing $A 1-\mathrm{KO}^{21}$ and $\mathrm{K} 2-\mathrm{KO}$ mice ${ }^{8}$. Embryonic day was estimated considering noon of the day of a vaginal plug as E0.5. Fetuses were collected at different embryonic stages (E) by cesarean section. Both A1-KO and $\mathrm{K} 2-\mathrm{KO}$ mice were generated in a mixed genetic background C57BL6/Sv129J and housed under diurnal lighting conditions (12 h dark/light). Experiments were performed according to the international guidelines for animal research. The experimental protocol was approved by the Animal Care Committee of the "Federico II" University of Naples. To assess the genotype of the mice, DNA was obtained from a small piece of tissue from the mouse. The tissue was incubated overnight at $60^{\circ} \mathrm{C}$ with lysis buffer (50 mM Tris- $\mathrm{HCl}, 100 \mathrm{mM}$ EDTA, $100 \mathrm{mM} \mathrm{NaCl}, 1 \%$ SDS, $0.5 \mathrm{mg} / \mathrm{ml}$ proteinase $\mathrm{K})$, and genomic DNA was extracted by adding 0.3 volumes of $6 \mathrm{M} \mathrm{NaCl}$ and precipitated with isopropyl alcohol. DNA concentration was determined using NanoDrop ND1000 (NanoDrop, Wilmington, DE), and equal amount of DNA was used for PCR analysis. The annealing temperature for Hmgal and Hipk2 alleles was $55^{\circ} \mathrm{C}$. The PCR products of Hmgal and Hipk2 were separated on a $1 \%$ or $2 \%$ agarose gel, respectively. Gels were scanned with Chemidoc (Bio-Rad, Hercules, CA). The following primers were used:

Hmga1-Fw 5'-AGAGACAAGAATGGGAGAGC-3' Hmgalwt-Re 5'-TGTTACTAGGACCCTCATGG-3' Hmga1KO-Re 5'-TAAAGCGACTGCTCCAGACT-3' Hipk2-Fw 5'-TAGTACCCAGGTGAACCTTGGAGT-3' Hipk2wt-Re 5'-GCTTCTCTCAAACTAAAGACCACG C-3'

\section{Hipk2KO-Re 5'-CAAAGGGTCTTTGAGCACCAGA-3'}

For Hmga1 gene, the wt allele was amplified using Hmga1-Fw + Hmgalwt-Re primers, and the knock-out allele was amplified using Hmga1-Fw + Hmga1KO-Re primers. For Hipk2 gene, the wt allele was amplified using Hipk2-Fw + Hipk2wt-Re primers, and the knock-out allele was amplified using Hipk2-Fw + Hipk2KO-Re primers. MEFs have been isolated and genotyped as already described $^{19,57}$. Animals were autopsied (Fondazione Filarete Per Le Bioscienze E L'innovazione), and all tissues were examined regardless of their pathological status. Only DKO which show perinatal death have been used for gene expression and IHC experiments.

\section{RNA extraction, RT-PCR, and quantitative RT-PCR}

Total RNA from E17.5 embryos, cell lines, and tissues of newborn puppies was extracted as already described $^{58}$. cDNA was synthesized from total RNA through reverse transcription, and subsequent PCR amplification was performed as previously described ${ }^{59}$. cDNA and qRT-PCR analysis for Ttf1, Pax8, Foxe1, Sp-C, Sp-A, $S p-B, T g, T p o$, and $T s h r$ was performed as already described ${ }^{58}$. The following primers were used for qRTPCR:

mouse- $T g$-Fw $\quad$ 5'-CATGGAATCTAATGCCAAGAAC TG-3' 
mouse- $T g$-Re 5' -TCCCTGTGAGCTTTTGGAATG-3' mouse- Tpo-Fw 5'-CAAAGGCTGGAACCCTAATTT CT-3'

mouse-Tpo-Re $\quad 5^{\prime}$-AACTTGAATGAGGTGCCTTGT

CA-3'

mouse-Tshr-Fw 5'-TCCCTGAAAACGCATTCCA-3' mouse-Tshr-Re 5'-GCATCCAGCTTTGTTCCATTG-3' mouse-Ttf1-Fw 5'-TTACCAGGACACCATGCGG-3' mouse-Ttf1-Re 5'-TGCCACTCATATTCATGCCG-3' mouse-Pax8-Fw 5'-GCCATGGCTGTGTAAGCAAGA-3' mouse-Pax8-Re 5'-GCTTGGAGCCCCCTATCACT-3' mouse-Foxe1-Fw 5'-AAGCCGCCCTACAGCTACATC G-3'

mouse-Foxe1-Re 5'-AACATGTCCTCGGCGTTGGG-3' mouse-Sp-C-Fw 5'-GGTCCTGATGGAGAGTCCAC-3' mouse-Sp-C-Re 5' -GATGAGAAGGCGTTTGAGGT-3' mouse-Sp-A-Fw 5'- CTGGAGAACATGGAGACAAG G-3'

mouse-Sp-A-Re 5' - AAGCTCCTCATCCAGGTAAGC-3' mouse-Sp-B-Fw 5' - AACCCCACACCTCTGAGAAC-3' mouse-Sp-B-Re 5' - GTGCAGGCTGAGGCTTGT-3' mouse-Actin-Fw 5'-CTAAGGCCAACCGTGAAAAG-3' mouse-Actin-Re 5'-ACCAGAGGCATACAGGGACA-3' To calculate the relative expression levels, the $2-\Delta \Delta C T$ method was used ${ }^{60}$.

The following primers were used for RT-PCR:

mouse-Hmga1-Fw 5'-GGCAGACCCAAGAAACTGG-3' mouse-Hmga1-Re 5'-GGCACTGCGAGTGGTGAT-3' mouse-Hipk2-Fw 5'-GAGACACAGGCTCAAGATGG-3' mouse-Hipk2-Re 5'-TCTGCTCGTAAGGTAGGCTT-3' mouse-G6pd-Fw 5'-GAAAGCAGAGTGAGCCCTTC-3' mouse-G6pd-Re 5'-CATAGGAATTACGGGCAAAGA-3' The primers used for Actin were above described.

\section{Protein extraction and western blotting}

Protein extraction and western blotting experiments were performed as already described ${ }^{61,62}$. The antibodies used were: anti- $\beta$-actin (sc-1616, Santa Cruz Biotechnology, Inc., Santa Cruz, CA), anti- $\gamma$-tubulin (sc-17787, Santa Cruz), anti-GAPDH (sc-32233, Santa Cruz), anti-vinculin (sc-7649, Santa Cruz), anti-PAX8 ${ }^{63}$, anti-NKX2.1 ${ }^{33}$, antiFOXE1 ${ }^{64}$, anti-SP-B (ab3430, Millipore), anti-SP-C (90716, Abcam), anti-HIPK2 (Novus Biologicals), antiHMGA $1^{65}$. Densitometric analyses of western blot were performed with Image J software.

\section{Histology and immunohistochemistry}

When not spontaneously dead, animals were killed by decapitation. Sections of neck containing thyroid gland and lungs were fixed overnight at $4{ }^{\circ} \mathrm{C}$ in $4 \%$ paraformaldehyde in phosphate-buffered saline (PBS), $\mathrm{pH} 7.2$, dehydrated through ethanol series, cleared in xylene and embedded in paraffin. For histological analysis, $7 \mathrm{~mm}$ sections were stained with hematoxylin and eosin (Sigma-
Aldrich, St. Louis, MO), according to the manufacturer's instructions. For immunohistochemical analysis, $7 \mathrm{~mm}$ sections were dewaxed by standard techniques. Heat treatment was performed for antigen retrieval in sodium citrate buffer, $\mathrm{pH} 6$, followed by permeabilization by incubating sections in PBS containing $0.2 \%$ Triton X-100. Incubation with primary antibodies was performed overnight at $4{ }^{\circ} \mathrm{C}$ in blocking buffer (3\% BSA, 5\% goat serum, $20 \mathrm{mM} \mathrm{MgCl} 2,0.3 \%$ Tween 20 in PBS). Endogenous peroxidase activity was quenched with $35 \% \mathrm{H}_{2} \mathrm{O}_{2}$ in methanol at room temperature, and chromogenic reactions were carried out according to the Vectastain $A B C$ kit protocol (Vector Laboratories, Burlingame, CA). No nichel was added in the chromogenic reaction for supplementary immunohistochemistry data. The following primary antibodies were used: anti-proSP-C (90716, Abcam); anti-SP-A (ab3420, Millipore); anti-SP-B (ab3430, Millipore); anti-PAX8; ${ }^{62}$ anti-NKX2.1;33 antiFOXE1; ${ }^{63}$ anti-TG rabbit polyclonal antibody (Dako). Biotinylated anti-rabbit IgG 1:200 was used for detection of primary antibodies (Vector Laboratories, Burlingame, CA).

\section{Cell cultures, transfections, and luciferase assays}

HeLa cells were grown in Dulbecco's modified Eagle's medium (Euroclone) supplemented with 10\% fetal bovine serum (Invitrogen). The pcDNA3.1-Hmga1b and pCEFLHA-Hipk2 vectors were previously described ${ }^{65,66}$. Cells were transfected as previously described ${ }^{67}$. In brief, HeLa cells were plated in six-well plates and after $24 \mathrm{~h}$ were transfected with $200 \mathrm{ng}$ of pLuc-Sp-C $\mathrm{C}^{34}$ with $50 \mathrm{ng}$ of pCMV-Renilla plasmid and with pCMV-TTF-1(Nkx2.1) expression vector ${ }^{35}$ alone or with different amount of pcDNA3.1-Hmga1b and pCEFL-HA-Hipk2 expression vectors.

BEAS-2B cells were grown in LHC-9 serum-free medium (Gibco, Invitrogen) supplemented with L-glutamine, retinoic acid, epinephrine, gentamicin, insulin, hydrocortisone, EGF, tranferrin, bovin pituitary extract, and T3 supplemented with RNAi was performed transfecting siRNA [HMGA1:Stealth siRNAs (Set of 3) HSS142459, HSS142460, HSS142461, Cat. \#1299001, (Life Technologies-Invitrogen); HIPK2: Stealth siRNAs (Set of 3) HSS120797, HSS120796, HSS178947, Cat. \#5258224. (Life Technologies-Invitrogen)] using Lipofectamine RNAiMAX (Thermofisher), according to manufacturer's instructions.

\section{Statistical analysis}

Data were analyzed using a two-sided unpaired Student's $t$ test (GraphPad Prism, GraphPad Software, Inc.). Values of $P<0.05$ were considered statistically significant ${ }^{68}$. All animal experiments included at least three biological replicates. Data are expressed as mean \pm SD. 


\section{Acknowledgements}

This work was supported by grants (MFAG-11702 to G.M.P. and IG-11477 to A.F.) from the Associazione Italiana Ricerca sul Cancro (AIRC), from the Italian Ministry of Economy and Finance to the CNR for the "Project FaReBio di Qualità", PNR-CNR Aging "Program 2012-2014".

\section{Author details}

'Department of Molecular Medicine and Medical Biotechnology, University of Naples, Naples, Italy. ${ }^{2}$ Institute of Endocrinology and Experimental Oncology, CNR, Naples, Italy. ${ }^{3}$ Present address: Institute of Experimental Genetics, Helmholtz Zentrum, Munich, Germany. ${ }^{4}$ Present address: Lymphocyte Nuclear Biology, NIAMS, NIH, Bethesda, MD, USA. ${ }^{5}$ Present address: AXXAM S.p.a.OpenZone, Bresso, Milan, Italy

\section{Authors' contributions}

R.G. and F.C. performed the mouse genetics and quantitative PCR experiments; A.C., C.G., V.V. and M.T. performed the cellular and molecular experiments; E.A., S.C. and S.P. performed immunohistochemical experiments; L.D.G. contributed to the mouse handling and genetics; G.D.V. contributed to critical review of the manuscript; G.M.P. and A.F. coordinated the work and wrote the manuscript. All the authors discussed the results and revised the manuscript text.

\section{Conflict of interest}

The authors declare that they have no conflict of interest.

\section{Publisher's note}

Springer Nature remains neutral with regard to jurisdictional claims in published maps and institutional affiliations.

Supplementary Information accompanies this paper at (https://doi.org/ 10.1038/s41419-019-1975-5).

Received: 16 December 2018 Revised: 3 September 2019 Accepted: 12 September 2019

Published online: 03 October 2019

\section{References}

1. Kim, Y. H., Choi, C. Y., Lee, S. J., Conti, M. A. \& Kim, Y. Homeodomain-interacting protein kinases, a novel family of co-repressors for homeodomain transcription factors. J. Biol. Chem. 273, 25875-25879 (1998).

2. D'Orazi, G., Rinaldo, C. \& Soddu, S. Updates on HIPK2: a resourceful oncosuppressor for clearing cancer. J. Exp. Clin. Cancer Res. 31, 63 (2012).

3. Schmitz, M. L., Rodriguez-Gil, A. \& Hornung, J. Integration of stress signals by homeodomain interacting protein kinases. Biol. Chem. 395, 375-386 (2014).

4. Conte, A. \& Pierantoni, G. M. Regulation of HIPK proteins by MicroRNAs. MicroRNA 4, 148-157 (2015).

5. Conte, A. \& Pierantoni, G. M. Update on the regulation of HIPK1, HIPK2 and HIPK3 protein kinases by microRNAs. MicroRNA 7, 178-186 (2018).

6. Isono, K. et al. Overlapping roles for homeodomain-interacting protein kinases hipk1 and hipk2 in the mediation of cell growth in response to morphogenetic and genotoxic signals. Mol. Cell Biol. 26, 2758-2771 (2006).

7. Zhang, J. et al. Essential function of HIPK2 in TGFbeta-dependent survival of midbrain dopamine neurons. Nat. Neurosci. 10, 77-86 (2007).

8. Anzilotti, S. et al. Genetic ablation of homeodomain-interacting protein kinase 2 selectively induces apoptosis of cerebellar Purkinje cells during adulthood and generates an ataxic-like phenotype. Cell Death Dis. 6, e2004 (2015).

9. Pierantoni, G. M. et al. High mobility group I (Y) proteins bind HIPK2, a serinethreonine kinase protein which inhibits cell growth. Oncogene 20, 6132-6144 (2001).

10. Manfioletti, G. et al. CDNA cloning of the HMGI-C phosphoprotein, a nuclear protein associated with neoplastic and undifferentiated phenotypes. Nucleic Acids Res. 19, 6793-6797 (1991).

11. Johnson, K. R., Lehn, D. A. \& Reeves, R. Alternative processing of mRNAs encoding mammalian chromosomal high-mobility-group proteins HMG-I and HMG-Y. Mol. Cell Biol. 9, 2114-2123 (1989).
12. Fusco, A. \& Fedele, M. Roles of HMGA proteins in cancer. Nat. Rev. Cancer $\mathbf{7}$ 899-910 (2007)

13. Pierantoni, G. M. et al. High-mobility group A2 gene expression is frequently induced in non-functioning pituitary adenomas (NFPAs), even in the absence of chromosome 12 polysomy. Endocr. Relat. Cancer 12, 867-874 (2005).

14. Fedele, M., Pierantoni, G. M., Visone, R. \& Fusco, A. Critical role of the HMGA2 gene in pituitary adenomas. Cell Cycle 5, 2045-2048 (2006).

15. Zhou, X., Benson, K. F., Ashar, H. R. \& Chada, K. Mutation responsible for the mouse pygmy phenotype in the developmentally regulated factor HMGI-C. Nature 376, 771-774 (1995).

16. Chiappetta, G. et al. Theexpression of the high mobility group HMGI (M) proteins correlates with the malignant phenotype of human thyroid neoplasias. Oncogene 10, 1307-1314 (1995).

17. Chiappetta, G. et al. HMGA2 mRNA expression correlates with the malignant phenotype in human thyroid neoplasias. Eur. J. Cancer 44, 1015-1021 (2008).

18. Pierantoni, G. M. et al. Deregulation of HMGA1 expression induces chromosome instability through regulation of spindle assembly checkpoint genes. Oncotarget 6, 17342-17353 (2015).

19. Pierantoni, G. M. et al. Hmga1 null mouse embryonic fibroblasts display downregulation of spindle assembly checkpoint gene expression associated to nuclear and karyotypic abnormalities. Cell Cycle 15, 812-818 (2016).

20. Conte, A. et al. High mobility group A1 protein modulates autophagy in cancer cells. Cell Death Differ. 24, 1948-1962 (2017).

21. Federico, A. et al. Hmga1/Hmga2 double knock-out mice display a "superpygmy" phenotype. Biol. Open 3, 372-378 (2014).

22. Diana, F. et al. Differential HMGA expression and post-translational modifications in prostatic tumor cells. Int J. Oncol. 26, 515-520 (2005).

23. Zhang, Q. \& Wang, Y. Homeodomain-interacting protein kinase-2 (HIPK2) phosphorylates HMGA1a at Ser-35, Thr-52, and Thr-77 and modulates its DNA binding affinity. J. Proteome Res. 6, 4711-4719 (2007).

24. Okuda, $\mathrm{H}$. et al. Novel interaction between HMGA1a and StP1 in murine terminally differentiated retina. Mol. Cell Neurosci. 33, 81-87 (2006).

25. Rinaldo, C. et al. HIPK2 controls cytokinesis and prevents tetraploidization by phosphorylating histone H2B at the midbody. Mol. Cell 47, 87-98 (2012).

26. Glasser, S. W., Baatz, J. E. \& Korfhagen, T. R. Surfactant protein-C in the maintenance of lung integrity and function. J. Aller Ther. S7, 001 (2011).

27. Pierantoni, G. M. et al. The homeodomain-interacting protein kinase 2 gene is expressed late in embryogenesis and preferentially in retina, muscle, and neural tissues. Biochem. Biophys. Res. Commun. 290, 942-947 (2002).

28. Chiappetta, G. et al. High level expression of the HMGl (Y) gene during embryonic development. Oncogene 13, 2439-2446 (1996).

29. Whitsett, J. A. \& Weaver, T. E. Hydrophobic surfactant proteins in lung function and disease. N. Engl. J. Med. 347, 2141-2148 (2002).

30. Mendelson, C. R. Role of transcription factors in fetal lung development and surfactant protein gene expression. Annu. Rev. Physiol. 62, 875-915 (2000).

31. Zhou, L., Lim, L., Costa, R. H. \& Whitsett, J. A. Thyroid transcription factor-1, hepatocyte nuclear factor-3beta, surfactant protein B, C, and Clara cell secretory protein in developing mouse lung. J. Histochem. Cytochem. 44, 1183-1193 (1996)

32. Kelly, S. E., Bachurski, C. J., Burhans, M. S. \& Glasser, S. W. Transcription of the lung-specific surfactant protein $C$ gene is mediated by thyroid transcription factor 1. J. Biol. Chem. 271, 6881-6888 (1996).

33. Lazzaro, D., Price, M., De Felice, M. \& Di Lauro, R. The transcription factor TTF-1 is expressed at the onset of thyroid and lung morphogenesis and in restricted regions of the fetal brain. Development 113, 1093-1104 (1991).

34. Park, K. S. et al. TAZ interacts with TTF-1 and regulates expression of surfactant protein-C. J. Biol. Chem. 279, 17384-17390 (2004).

35. Bohinski, R. J., Di Lauro, R. \& Whitsett, J. A. Lung-specific surfactant protein B gene promoter is a target for thyroid transcription factor 1 and hepatocyte nuclear factor 3 indicating common mechanisms for organ-specific gene expression along the foregut axis. Mol. Cell Biol. 14, 5671-5681 (1994).

36. Bruno, M. D., Bohinski, R. J., Huelsman, K. M., Whitsett, J. A. \& Korfhagen, T. R. Lung cell-specific expression of the murine surfactant protein A (SP-A) gene is mediated by interaction between the SP-A promoter and thyroid transcription factor-1. J. Biol. Chem. 270, 6531-6536 (1995).

37. Whitsett, J. A. \& Alenghat, T. Respiratory epithelial cells orchestrate pulmonary innate immunity. Nat. Immunol. 16, 27-35 (2015).

38. Speer, C. P. Neonatal respiratory distress syndrome: an inflammatory disease? Neonatology 99, 316-319 (2011).

39. Morrisey, E. E. \& Hogan, B. L. Preparing for the first breath: genetic and cellular mechanisms in lung development. Dev. Cell 18, 8-23 (2010). 
40. Whitsett, J. A. \& Glasser, S. W. Regulation of surfactant protein gene transcription. Biochim. Biophys. Acta 1408, 303-311 (1998).

41. Toledo, F. \& Wahl, G. M. Regulating the p53 pathway: in vitro hypotheses, in vivo veritas. Nat. Rev. Cancer 6, 909-923 (2006).

42. Glasser, S. W. et al. Altered stability of pulmonary surfactant in SP-C-deficient mice. Proc. Natl Acad. Sci. USA 98, 6366-6371 (2001).

43. Wert, S. E., Whitsett, J. A. \& Nogee, L. M. Genetic disorders of surfactant dysfunction. Pediatr. Dev. Pathol. 12, 253-274 (2009).

44. Nardinocchi, L., Puca, R., Sacchi, A. \& D'Orazi, G. Inhibition of HIF-1alpha activity by homeodomain-interacting protein kinase-2 correlates with sensitization of chemoresistant cells to undergo apoptosis. Mol. Cancer 8, 1 (2009).

45. Saini, Y., Harkema, J. R. \& LaPres, J. J. HIF1a is essential for normal intrauterine differentiation of alveolar epithelium and surfactant production in the newborn lung of mice. J. Biol. Chem. 283, 33650-33657 (2008).

46. Cheong, $\mathrm{H}$. et al. Analysis of a lung defect in autophagy-deficient mouse strains. Autophagy 10, 45-56 (2014).

47. Santisteban, P., Acebrón, A., Polycarpou-Schwarz, M. \& Di Lauro, R. Insulin and insulin-like growth factor I regulate a thyroid-specific nuclear protein that binds to the thyroglobulin promoter. Mol. Endocrinol. 6, 1310-1317 (1992).

48. Zannini, M., Francis-Lang, H., Plachov, D. \& Di Lauro, R. Pax-8, a paired domaincontaining protein, binds to a sequence overlapping the recognition site of a homeodomain and activates transcription from two thyroid-specific promoters. Mol. Cell Biol. 12, 4230-4241 (1992).

49. Minoo, P. et al. TTF-1 regulates lung epithelial morphogenesis. Dev. Biol. 172 694-698 (1995).

50. Civitareale, D., Castelli, M. P., Falasca, P. \& Saiardi, A. Thyroid transcription factor 1 activates the promoter of the thyrotropin receptor gene. Mol. Endocrinol. 7, 1589-1595 (1993).

51. Civitareale, D., Lonigro, R., Sinclair, A. J. \& Di Lauro, R. A thyroid-specific nuclear protein essential for tissue-specific expression of the thyroglobulin promoter. EMBO J. 8, 2537-2542 (1989)

52. Francis-Lang, H., Price, M., Polycarpou-Schwarz, M. \& Di Lauro, R. Cell-typespecific expression of the rat thyroperoxidase promoter indicates common mechanisms for thyroid-specific gene expression. Mol. Cell Biol. 12, 576-588 (1992).

53. De Felice, M. et al. TTF-1 phosphorylation is required for peripheral lung morphogenesis, perinatal survival, and tissue-specific gene expression. J. Biol. Chem. 278, 35574-35583 (2003).
54. Yan, C., Sever, Z. \& Whitsett, J. A. Upstream enhancer activity in the human surfactant protein B gene is mediated by thyroid transcription factor 1. J. Biol. chem. 270, 24852-24857 (1995).

55. Zannini, M. et al. Mapping and functional role of phosphorylation sites in the thyroid transcription factor-1 (TTF-1). J. Biol. Chem. 271, 2249-2254 (1996).

56. Whitmarsh, A. J. \& Davis, R. J. Transcription factor AP-1 regulation by mitogenactivated protein kinase signal transduction pathways. J. Mol. Med. (Berl.) 74, 589-607 (1996).

57. Valente, D. et al. HIPK2 deficiency causes chromosomal instability by cytokinesis failure and increases tumorigenicity. Oncotarget $\mathbf{6}$ 10320-10334 (2015).

58. Esposito, F. et al. HMGA1 pseudogenes as candidate proto-oncogenic competitive endogenous RNAs. Oncotarget 5, 8341-8354 (2014).

59. Pierantoni, G. M. et al. A truncated HMGA1 gene induces proliferation of the 3T3-L1 pre-adipocytic cells: a model of human lipomas. Carcinogenesis 24, 1861-1869 (2003).

60. Livak, K. J. \& Schmittgen, T. D. Analysis of relative gene expression data using real-time quantitative PCR and the 2(-Delta Delta C(T)) method. Methods 25, 402-408 (2001).

61. Conte, A. et al. Effects of resveratrol on p66Shc phosphorylation in cultured prostate cells. Transl. Med. UniSa 13, 47-58 (2016).

62. Conte A. et al. Convergent effects of resveratrol and PYK2 on prostate cells. Int J. Mol. Sci. 2016; 17, pii: E1542 (2016).

63. Amendola, E. et al. A mouse model demonstrates a multigenic origin of congenital hypothyroidism. Endocrinology 146, 5038-5047 (2005).

64. Dathan, N., Parlato, R., Rosica, A., De Felice, M. \& Di Lauro, R. Distribution of the titf2/foxe1 gene product is consistent with an important role in the development of foregut endoderm, palate, and hair. Dev. Dyn. 224 450-456 (2002).

65. Pierantoni, G. M. et al. High-mobility group A1 proteins are overexpressed in human leukaemias. Biochem J. 372(Pt 1), 145-150 (2003).

66. Hofmann, T. G. et al. Regulation of p53 activity by its interaction with homeodomain-interacting protein kinase-2. Nat. Cell Biol. 4, 1-10 (2002).

67. Valentino, T. et al. PATZ1 interacts with p53 and regulates expression of p53target genes enhancing apoptosis or cell survival based on the cellular context. Cell Death Dis. 4, e963 (2013).

68. Vitale, N. et al. Resveratrol couples apoptosis with autophagy in UVB-irradiated HaCat cells. PLoS ONE 8, e80728 (2013). 